\title{
FASES EN LA DERIVACIÓN E INTERPRETACIÓN SEMÁNTICO-PRAGMÁTICA
}

\author{
Lucia Alabart Lago luciaalabartlago@gmail.com \\ Guadalupe Herrera g.s.v.herrera@gmail.com \\ Daniel Romero daniel.dromakd@gmail.com \\ Centro de Estudios e Investigaciones Lingüísticas - Facultad de \\ Humanidades y Ciencias de la Educación - UNLP
}

\begin{abstract}
Resumen: En este trabajo se busca elaborar una descripción de la interfaz sintaxis-significado a partir de la articulación de propuestas relevantistas y generativas. En particular, se propone que las operaciones de extracción de inferencias de la Teoría de la Relevancia (TR) se aplican durante la derivación sintáctica, independientemente de que se haya transferido o no una fase. Partiendo de la idea de un paralelismo entre ambos procesos, se intenta demostrar que algunos mecanismos inferenciales imponen condiciones que afectan la valoración de los rasgos de las categorías funcionales. Además, se considera que $\mathrm{D}$ es núcleo de fase porque la valoración de rasgos de otros núcleos, como los de $\mathrm{T}$, en el curso de la derivación dependen crucialmente de los valores que adquieren los rasgos en D [Para propuestas de D como núcleo de fase puede verse Raposo (1999), Svenonious (2002), Boskovik (2008), Romero (2008, 2010) y Embick (2010)].
\end{abstract}

Palabras clave: fase - categorías funcionales - inferencia.

\begin{abstract}
This paper analyzes two texts produced by them, and reflect on how, in this context of crisis, the language is set as the possibility of rearticulation of an area of the common. This work is aimed at describing the syntax-meaning interface in terms of the interaction between relevantist and generative proposals. Our proposal is that the pragmatic operations which yield inferences put forward in Relevance Theory (RT) apply during the syntactic derivation and independently of phase transfer in the syntax. Taking the parallelism proposed in RT between these two processes, we will try to show that some inferential operations impose conditions on the functional elements, affecting their features valuation. We also support the view that $\mathrm{D}$ is a phase head, basing this idea on the fact that feature valuation of other heads, such as $\mathrm{T}$, crucially depends on the values of the features in $\mathrm{D}$.
\end{abstract}

Key words: phase - functional category - inference. 


\section{Introducción}

En trabajos anteriores (Romero, 2008a y b; Alabart Lago, L., P. Díaz y G. Herrera, 2012; Alabart Lago, L. y Herrera, G; 2013) se intentó mostrar que el mecanismo interpretativo que propone la Teoría de la Relevancia (TR) podría resultar adecuado como uno de los sistemas externos (también interpretativos) postulados por la Gramática Generativa (GG), más precisamente, el sistema llamado Conceptual-Intencional (CI). En este sentido, Leonetti y Escandell Vidal (2004) sostienen que las categorías funcionales (CCFF) de la GG pueden considerarse equivalentes a las categorías procedimentales (CCPP) propuestas por la TR.

La TR (Sperber y Wilson, 1986) afirma que la interpretación de un enunciado se deriva de las estructuras sintácticas, y se realiza "en paralelo" con la derivación de estructuras llevada a cabo por las operaciones del componente sintáctico; así lo explicita Recanati (2010):

According to relevance theory, pragmatic processing take us from the gappy mental representations associated with sentences in virtue of the semantic rules of the language to full-fledged mental representations, namely the thoughts which the contextualized utterances express (p. 232).

Por otro lado, y en relación a cómo procede la interpretación de enunciados, tenemos en cuenta que en Chomsky (2008) se dejan de lado los niveles de representación internos - la estructura profunda (EP) y la superficial (ES) - y se considera prescindible la interfaz Forma Lógica (FL), de 
modo que las estructuras generadas se transfieren a los sistemas externos en cuanto los rasgos formales de las CCFF son valorados.

En relación a lo anteriormente mencionado, nuestra hipótesis puede resumirse en lo siguiente: las operaciones de extracción de inferencias propuestas por TR se aplican durante la derivación sintáctica independientemente de que se haya transferido o no una fase. Pretendemos mostrar también que algunos mecanismos inferenciales imponen ciertas condiciones que afectan a la valoración de los rasgos de algunas CCFF, como el tiempo (T) y los determinantes (D), que son "híbridos" (Carston, 2009; y Alabart Lago y Herrera, 2013). Este fenómeno es una de las pruebas que nos llevan a postular que el módulo interpretativo accede de forma relativamente "irrestricta" a la derivación sintáctica.

Con estos fundamentos esperamos poder elaborar una descripción de cómo interactúan ambos sistemas en la derivación de una oración y la asignación (casi simultánea) de significado.

Nuestro trabajo abordará las siguientes cuestiones:

En la primera sección, se analizarán la noción de fase en la GG y algunas cuestiones importantes respecto del dudoso estatus de las categorías D y T como núcleos de fase. En la segunda sección, se abordarán las razones para la eliminación de FL en la GG y en la TR, ya que en Chomsky (2008) se plantea que ya no existe este nivel de interfaz, por lo tanto, es necesario establecer si es posible eliminarlo además como nivel de representación en la TR. Luego se introducirán algunos 
presupuestos de la TR y la relación entre las CCPP y las CCFF, propuesta por Leonetti y Escandell-Vidal (2004), en la que nos basamos; y finalmente, se retomarán cuestiones previamente esbozadas acerca del estatus de $\mathrm{T}$ y $\mathrm{D}$ como núcleos de fase y algunos problemas teóricos que se generan y que indican que el módulo interpretativo opera mediante el "acceso relativamente irrestricto" a la estructura sintáctica. En la última sección del trabajo, se explicitarán las consecuencias derivadas de nuestra hipótesis y algunos problemas o cuestiones a resolver.

\section{Derivación por fases y transferencia}

La idea que subyace a la derivación por fases no es novedosa, se propuso por primera vez en Chomsky, Halle y Lakoff (1956) como un criterio para ordenar las reglas de acentuación en fonología, con la denominación de ciclo. Posteriormente Chomsky (1966) extiende esta noción a las reglas transformacionales; el Principio de Ciclicidad postula que los movimientos de constituyentes se aplican "de abajo hacia arriba", es decir, desde la oración más incrustada para terminar en la matriz. Más adelante, en el marco de Principios y Parámetros ( $\mathrm{P}$ y $\mathrm{P}$ ), la noción de ciclo se sustituye por la de barreras (también nudo limitante), como en Chomsky (1986).

Las fases se definen como derivaciones parciales, afectan a un subconjunto de los elementos léxicos y funcionales comprendidos en una derivación y limitan los Ensambles Internos ${ }^{1}$; los rasgos no interpretables de los constituyentes se

1 Nos referimos a la operación más fundamental del sistema computacional de acuerdo con los postulados minimalistas: la operación que combina dos ítems léxicos para 
cotejan, reciben un valor y se eliminan; concluido el cotejo de rasgos, la fase es transferida a los niveles de interfaz. Chomsky (1998) propone la Condición de Impenetrabilidad de Fase (CIF): En la fase $\mathrm{F}$, con núcleo $\mathrm{H}$, el dominio de $\mathrm{H}$ no es accesible para las operaciones fuera de F, sólo $\mathrm{H}$ y su borde ${ }^{2}$.

Esta condición impone que los elementos que deben cotejar rasgos con un núcleo funcional deben estar en un dominio más restringido, el especificador de la fase previa "más externo" 3.

La noción de fase se especifica aun más en Chomsky (2001) con dos propuestas. En primer lugar, se redefine Materialización (Spell out). En Chomsky (1995), Materialización es una operación que separa los rasgos fonéticos y los envía a la interfaz Forma Fonética (FF), con el objetivo de reducir el costo de las operaciones posteriores. En Chomsky (2001), la operación Transferencia envía todos los rasgos de la fase ${ }^{4}$, tanto fonéticos como semánticos, a los componentes interpretativos ${ }^{5}$.

formar frases. El Ensamble se llama Interno para distinguirlo de una operación compuesta, denominada Ensamble Externo, que consta de dos suboperaciones Copiado y Ensamble del elemento copiado. Esta operación compuesta es lo que también se conoce como Movimiento y queda ilustrada por las interrogativas totales, por ejemplo: "¿[Qué mural] pintó tu amiga [Svpintar [SVpint- [qu- mural]]]]?

2 El término "borde" es traducción de "edge"; se denomina así a la posición de Especificador más externa de la derivación.

3 Tampoco la CIF es novedosa; en Chomsky (1973) el Ppio. de Ciclicidad se complementa con la Condición de Ciclo Estricto; que postula que los procesos transformacionales no pueden afectar a dominios más incrustados de la derivación una vez que han alcanzado un dominio superior. La CIF tendría la sola modificación de que permite acceso al borde o arista de la fase.

4 Salvo, por supuesto, los del núcleo y su borde, si estos deben ser cotejados.

5 No queda claro si los rasgos son transferidos a las interfaces, dado que posteriormente en Chomsky (2008) se considerará FL como prescindible. 
En segundo lugar, se propone que solo dos CCFF, el Complementante $(\mathrm{C})$ y el verbo liviano $(\underline{\mathrm{v}})$, son núcleos de fase "fuertes". Independientemente, en Chomsky (1998) se encuentra la sugerencia de que $\mathrm{D}$ también constituiría una fase. Esta CF no se menciona explícitamente en trabajos posteriores, pero se puede suponer que también $\mathrm{D}$ sería un núcleo de fase y que en su dominio se produce cotejo y valuación de rasgos mediante ensamble interno. De hecho, los sustantivos $(\mathrm{NN})$ tienen un conjunto de rasgos formales $\left(\right.$ rasgos $\left.^{\Phi}\right)$ que deben ser cotejados y evaluados por una CF, el D. Sin embargo, más adelante demostraremos que el cotejo y valuado de los rasgos [+/- definido] o [+/-específico] no depende sólo de $\mathrm{D}$ sino que es necesario esperar a que se ensamble T para establecer la "saturación", que a su vez es un proceso que realiza el módulo interpretativo.

Boeckx y Grohmann (2004) cuestionan algunas de las afirmaciones de Chomsky acerca de las fases. Consideran que la derivación por fases tiene muchos defectos similares a la noción de barrera de Chomsky (1986) y critican fundamentalmente la postulación de que $\mathrm{T}$ no posee rasgos que le permitirían ser sonda (probe) o posición de cotejo. Chomsky (2008) sostiene que los rasgos de T se "heredan" de C, noción muy similar a la de "barrera por herencia" de Chomsky (1986). Esta noción introdujo muchas dificultades en el modelo de Principios y Parámetros y fue en parte dejada de lado cuando se favoreció la "minimalidad relativizada" propuesta por Rizzi (1990). 
Por otra parte, en algunos trabajos y en la tesis doctoral de Ángel Gallego (2006 y 2007) ${ }^{6}$ y en Gallego y Uriagereka (2006) se demuestra con argumentos plausibles que, al menos para algunas lenguas, entre las que se cuenta al español, $T$ es un núcleo de fase fuerte, necesario para evaluar los rasgos no interpretables de algunos sintagmas determinante (SSDD) ensamblados con el verbo léxico (V) y $\underline{v}$.

\section{La noción de forma lógica}

\section{FL en GG}

En la GG, la FL se propone como nivel de representación en Chomsky (1976 y 1981) y May (1977). Poco tiempo después May (1985) establece el contenido y operaciones de FL cuando publica su tesis doctoral. FL es una estructura sintáctica que economiza el costo cognitivo de la interpretación semántica; comprende las estructuras de operadores y variables de distinto tipo (cuantificación, interrogación, negación), las relaciones de correferencia entre expresiones nominales (ligamiento) y las condiciones veritativas. Esta estructura se deriva de la ES mediante Mover- $\alpha$ restringida por condiciones similares a las que actúan en la sintaxis (subyacencia, Principio de categorías vacías, barreras).

Posteriormente, cuando Chomsky (1993 y 1995) formula el Programa Minimalista (PM) se eliminan por razones de economía computacional los niveles de representación "internos", EP y ES, y se mantienen los niveles de FF y FL

6 Gallego (2010) abandona esta postura y vuelve a que los únicos núcleos de fase son C y v. 
como niveles de interfaz, estructuras que deben ser "leídas" por otros sistemas que interactúan con la Facultad del Lenguaje. FF se relaciona con el sistema Sensorio-motriz (SM), que hace posible la pronunciación y percepción de expresiones lingüísticas, y FL con el sistema Conceptual-Intencional (CI), que interpreta el significado. Más adelante, Chomsky (2008) afirma que FL no es una interfaz, sino un nivel de representación "interno" porque se deriva con operaciones similares a las EP y ES. Asumiendo que la única operación que forma estructuras sintácticas es Ensamble (Merge), su propuesta es: "This computational architecture, if sustainable, seems to be about as good as possible. S-Structure and LF are no longer formulable as levels, hence disappear along with DStructure, and computations are reduced to a single cycle." (Chomsky, 2008, pág. 18) ${ }^{7}$.

En consecuencia, en GG, no habría FL, y surge el problema de determinar de qué manera se relacionan el sistema lingüístico en sentido estricto (la sintaxis) y el sistema externo CI.

\section{FL en TR}

Según Sperber y Wilson (1986) las FFLL son fórmulas bien formadas (susceptibles de ser derivadas sintácticamente) que pueden someterse a operaciones lógicas formales determinadas por su estructura (pueden implicarse y contradecirse mutuamente). Como son fórmulas, pueden estar almacenadas en la memoria conceptual como esquemas de supuesto

7 Esta arquitectura de las computaciones, si es sostenible, parece ser la mejor posible. ES y FL ya no son formulables como niveles, por lo tanto, desaparecen junto con la Estructura-P, y las computaciones se reducen a un solo ciclo. (nuestra traducción). 
similares a los de la lógica proposicional (Si P entonces $Q$, por ejemplo). Sin embargo, no son representaciones semánticas en sentido estricto sino descripciones estructurales de expresiones lingüísticas.

Las FFLL son representaciones que el sistema lingüístico entrega y a partir del cual el módulo interpretativo opera para obtener las representaciones semánticas o Formas Proposicionales (FFPP). Las FFPP, entonces, son definidas como formas lógicas semánticamente completas, que pueden evaluarse como verdaderas o falsas y constituyen conocimientos enciclopédicos.

Según Recanati (2010), las FFLL son el output de la decodificación lingüística y no son semánticamente evaluables porque son demasiado indeterminadas. El módulo interpretativo opera con esas FFLL completándolas mediante distintos procesos (de saturación, enriquecimiento y aflojamiento, asignación de referencia y fuerza ilocutoria, entre otros) para obtener una forma proposicional (FP), que constituye una representación semántica. Por lo tanto, las FFPP se consideran como constructos que resultan legibles tanto en la sintaxis como en el módulo interpretativo y constituyen la interfaz entre el sistema lingüístico y el de pensamiento (Recanati, 2010).

Para Sperber y Wilson ni las condiciones veritativas ni las propiedades lógicas de alcance de cuantificadores y las dependencias anafóricas se resuelven en la FL sino en la FP o 
explicatura $^{8}$ (nivel posterior que sigue a los procesos de saturación, enriquecimiento y aflojamiento). Por lo tanto, consideramos que no hay necesidad de postular que la FL sea un nivel de representación: el módulo interpretativo opera en simultáneo con la derivación sintáctica, como se mostrará más abajo.

\section{Unidades conceptuales y unidades procedimentales}

Siguiendo las concepciones pragmáticas fundamentales de la TR, Escandell-Vidal y Leonetti (2004) proponen que para estudiar el proceso de interpretación de enunciados conviene, teórica y empíricamente, concebir que el aporte que hacen las unidades lingüísticas a la interpretación es doble: conceptual y procedimental. Se supone entonces la existencia de dos semánticas: una aporta representaciones conceptuales y la otra proporciona instrucciones de procesamiento, es decir, indica cómo combinar esas representaciones. Dada esa distinción, se consideran procedimentales las unidades lingüísticas que codifican información referencial de tiempo, lugar y entidades (expresiones referenciales), los marcadores del discurso, los mecanismos sintácticos que determinan la estructura informativa (topicalización/focalización), "las marcas de modalidad, las partículas citativas y evidenciales, la entonación, los tiempos y modos verbales, los determinantes, y los adverbios deícticos y focalizadores". (Leonetti y EscandellVidal, 2004, p.1).

8 En TR se consideran dos clases de explicaturas, las explicaturas proposicionales, sobre las que se establecen las condiciones veritativas y las explicaturas de nivel superior, sobre las que se interpretan la modalidad y fuerza ilocutoria. 
Por lo tanto, las unidades procedimentales restringen las explicaturas, proposicionales y de nivel superior, y las implicaturas, dando instrucciones al módulo pragmático respecto de la asignación de referentes y las posibilidades ilocutivas del enunciado. La interpretación de unidades conceptuales requiere el acceso no sólo al conocimiento enciclopédico (lexicón), sino también al conocimiento del mundo para "asociar palabras con entidades, situaciones o hechos extralingüísticos". (Leonetti y Escandell-Vidal, 2004, p. 4).

La relación entre las unidades lingüísticas y el contexto va en direcciones opuestas para los elementos de una u otra semántica. Por un lado, los conceptos son susceptibles al contexto, que puede requerir que se interpreten de forma más específica o más abarcadora. El reajuste y la coacción son fenómenos comunes y sistemáticos en la comunicación porque el contenido conceptual es flexible y adaptable.

(1) a. ¿Cortamos la torta?

b. Lucía la cortó con el novio.

c. Salieron juntos pero Juan se cortó solo y se fue a otro bar.

(2) Entonces supo que le había mentido.

En los ejemplos de (1) se da el fenómeno de reajuste conceptual; el verbo <cortar>, debido a que aparece en distintas configuraciones sintácticas reajusta su contenido conceptual. 
Por otro lado, es posible interpretar (2) como En ese momento se percató de que le había mentido, atendiendo al proceso de coacción, por el cual se resuelven los conflictos entre el aspecto léxico (Aktionsart) del verbo "saber" y el aspecto gramatical (perfectivo). Como se evidencia en este ejemplo, es posible ver que la coacción se resuelve en pro del contenido procedimental. Es decir que se satisface la instrucción procedimental a costa del reajuste conceptual.

El contenido procedimental, entonces, es rígido y, así como obliga a reajustar el contenido conceptual, también impone condiciones al contexto de manera que se requiere la inserción de supuestos para satisfacer las instrucciones de procesamiento, como puede verse en (3):

(3)Cuidado con el perro.

En este caso, el D definido exige la recuperación de un referente, dado que no hay ninguno presente en el discurso previo, la semántica procedimental exige construir una nueva representación, en este caso una del tipo de existe un perro.

\section{Algunas reflexiones sobre el estatus de $\mathbf{T}$ y $\mathbf{D}$}

En adelante asumimos como correctas dos nociones:

Que C, v y D son los únicos núcleos de fase, y, por lo tanto, los dos estadios de la derivación en los que se aplica Transferencia. 
Que la noción de FL de la TR es irrelevante como nivel interpretativo, dado que: a) no se define formalmente ni se establece con claridad qué procesos inferenciales se aplicarían sobre esta estructura; b) al postular que la interpretación semántico-pragmática se da "en paralelo" con la derivación sintáctica, proponer una representación sobre la cual actuaría el módulo interpretativo resulta innecesario; c) el componente computacional no requiere de FL (de acuerdo a Chomsky, 2008) y, en consecuencia, hay solo una interfaz, $\mathrm{FF}^{9}$. De todas maneras, Chomsky (2008) menciona en varios párrafos que el contenido de una fase se transfiere a la interfaz con CI, de lo cual se puede inferir que habría un nivel de interfaz no definido sobre el que se realizarían las operaciones interpretativas. Podemos agregar que, si no existiera un nivel de interfaz en que pueda aplicarse la CIF a los rasgos semánticos. resultaría un poco confuso determinar cómo CI impone sus condiciones de legibilidad.

Transferencia, como se ha dicho, es una operación que permite reducir el costo de los procesos computacionales. Pero que los rasgos semánticos sean enviados a CI (o a alguna interfaz) no es un obstáculo para que los procesos interpretativos accedan a la derivación sintáctica en un estadio distinto de aquel en que se aplica Transferencia.

En este sentido, consideraremos algunos casos en los que es posible hipotetizar que el sistema interpretativo debe tener acceso a la derivación sintáctica para legitimar rasgos de los constituyentes.

9 Puede suponerse que FF sobrevive por la necesidad de aplicar ciertas operaciones como la linealización y la eliminación de copias. 


\section{Rasgos de D}

Consideremos los siguientes ejemplos:

(4) ¿De cuál de los pintores impresionistas viste un cuadro en el museo?

(5) * ¿De cuál de los pintores impresionistas viste este cuadro en el museo?

(6)

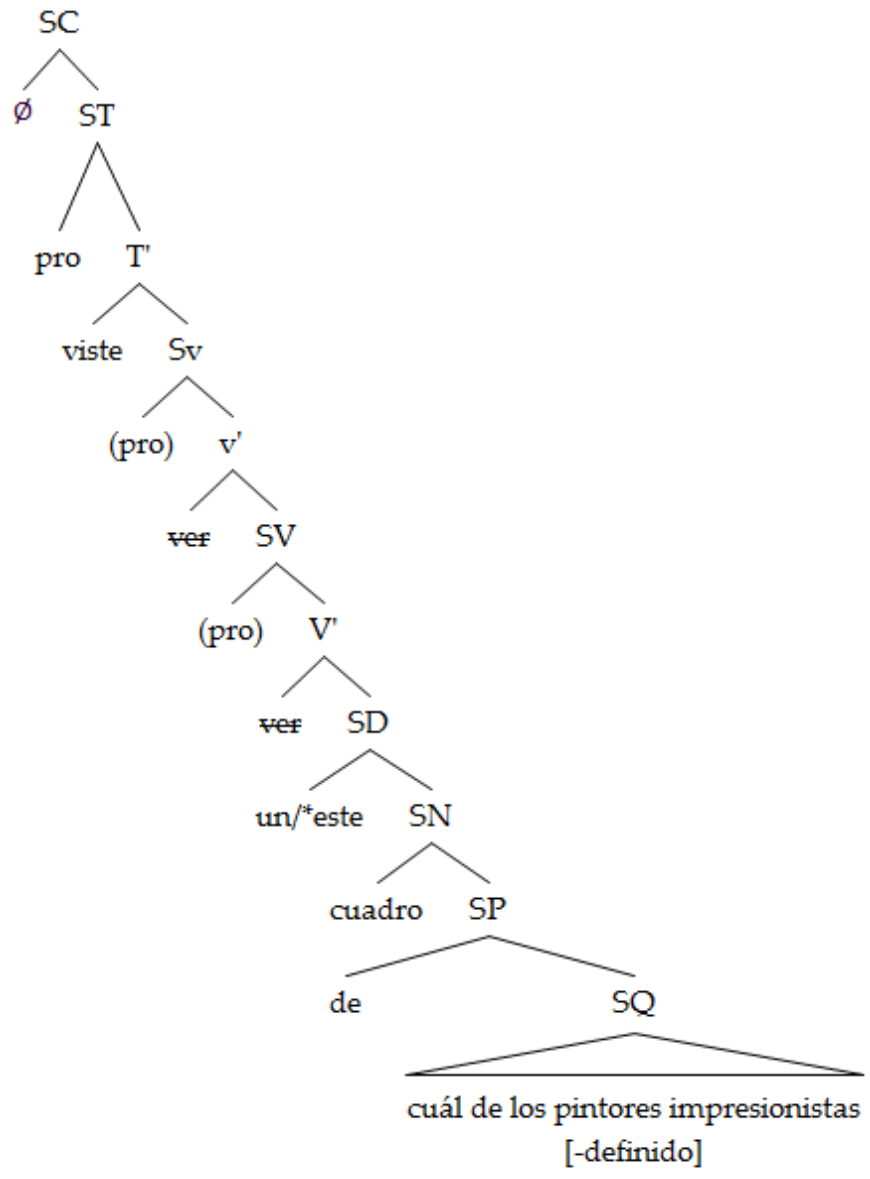


Ambos ejemplos tienen la siguiente estructura luego de la aplicación de Ensamble Interno al sintagma de cuantificador (SQU).

(7) ¿[De cuál de los pintores impresionistasi $]$ viste un/*este cuadro hi10en el museo?

El ejemplo (6) puede bloquearse si se acepta un principio que Huang (1994) propone para la FL:

Un SN [+definido] no puede contener variables.

La huella o copia del SQU desplazado es precisamente una variable ligada por el SQU. El inconveniente es que, si no existe el nivel de FL, el principio citado no puede aplicarse.

Observemos las siguientes estructuras de los SSDD contenidos en (8) y (9):

(8) Este cuadro de un pintor impresionista.

(9) ? Este cuadro de cuál de los pintores impresionistas.

El SD (9) resulta poco aceptable, dado que el $\mathrm{N}$ cuadro selecciona un complemento, al que asigna un papel temático ${ }^{11}$,

10 Usamos la notación típica del modelo de PyP para facilitar la exposición, En términos minimalistas, hi es la copia 'baja'-no pronunciada- del SP de qu- de los pintores impresionistas que, por motivos de linerización (Kayne, 1994), no se materializa fonológicamente. 
por lo que la expresión que se ensamble como complemento debe ser necesariamente referencial. Un SQU puede ser referencial y recibir papel temático, pero su referencia no es definida ni específica. Si $\left[ \pm\right.$ definido] o $[ \pm \text { específico }]^{12}$ son rasgos formales de deben cotejarse y valorarse con un elemento léxico, un $\mathrm{N}$, la estructura (3) sería una derivación fallida (no convergente) porque de acuerdo la CIF el rasgo no es legítimo para CI.

Ahora bien, la estructura de (4) se transfiere en la última fase, con el Ensamble de $\mathrm{C}^{13}$. Obsérvese en la representación (6) que, al ensamblarse el D este con el SN cuadro de cuál de los pintores impresionistas, hay rasgos que no pueden ser cotejados y valorados ${ }^{14}$. Sin embargo, la derivación no se cancelaría hasta el ensamble de $\mathrm{C}$, dado que, en cualquier instancia previa de ensamble, restarían varios rasgos sin cotejo ([Caso], [tiempo], [ $\pm \mathrm{QU}]$, etc.), de modo que la Transferencia se efectuaría recién cuando se agotara la última fase - SC. Es decir, la sintaxis debe arrastrar un conjunto léxico que va inevitablemente a fallar en la interfaz con CI.

\section{Rasgos de T}

Consideremos:

11 Dejamos aparte la cuestión de si los papeles temáticos son asignados a los argumentos o rasgos de los elementos léxicos que deben ser cotejados y valorados. En este caso el PT puede ser Agente, Poseedor, etc.

12 Huang (1994) no considera diferencias entre [ \pm definido] o [ \pm específico], tampoco entre SN y SD.

13 Que no se pronuncia (ø) pero coteja el rasgo qu- del SP de qu- de los pintores impresionistas y se ensambla con la copia 'alta' del SP, una vez que el rasgo qu- está valuado.

14 Por ejemplo, el rasgo qu- del SP de qu-de los pintores impresionistas. 
(10) Te aconsejo que te acuestes.

(11) ? Te aconsejo que te hayas acostado (ayer).

La oración (11) es anómala. De acuerdo a Searle (1975) violaría la regla o condición de contenido proposicional ${ }^{15}$ :

Un acto ilocutorio directivo debe contener un acto futuro del oyente.

Veamos primeramente la estructura básica de (11):

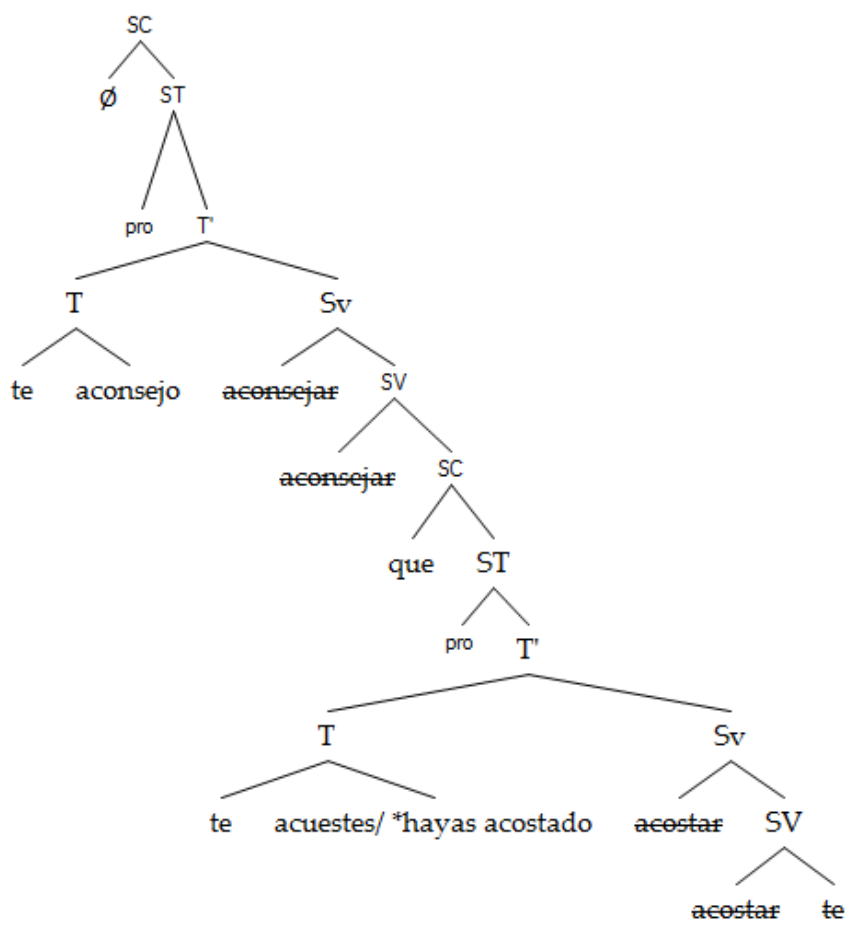

15 Las condiciones/reglas de los actos ilocutorios son particulares para cada tipo de acto, hay actos que no tendrían contenido proposicional definido, por ejemplo, las felicitaciones.

N 7. Primer Semestre de 2017 
Los rasgos de T se "heredan" de C (Chomsky, 2008). Supongamos que la selección de aconsejar se satisface por Ensamble Externo, es decir, por ser un $\mathrm{V}$ ilocutorio con determinado significado, selecciona determinados rasgos de su complemento, el SC. De esto se sigue que C debería tener rasgos de tiempo para que $\mathrm{T}$ los "herede". Entonces surgen algunas cuestiones complejas:

(a) ¿Cómo se satisfacen? Supongamos que fuera por Concordancia a Distancia. Si es un rasgo del V aconsejar es un elemento léxico, no funcional, no puede ser sonda; asimismo, no es muy claro cuál sería la meta. Si aceptamos que $C$ posee rasgos de tiempo, podría ser meta, pero la relación de cotejo/concordancia se establecería a la inversa, es decir, de una categoría léxica (sonda) a una categoría funcional (meta). Suñer y Padilla (1987) exploran la relación entre las esferas temporales a las que pertenecen los verbos principal y subordinado, proponiendo una combinatoria para los tiempos verbales de $\mathrm{V}_{1}$ - el verbo principal - y $\mathrm{V}_{2}$ - el verbo subordinado - en la que las restricciones léxicas que imponen los predicados se conciben en términos de coordinación entre las esferas ${ }^{16}$

(b) De acuerdo a Austin (1964) un enunciado realizativo debe estar en presente de indicativo, como se ilustra en (13):

16 Los autores distinguen las siguientes clases semánticas de verbos: de negación, emotivos-factivos, dubitativos, de influencia, desiderativos y de falta de conocimiento. Los primeros, de acuerdo con los autores, son los que menos restricciones presentan; admiten todas las combinaciones posibles entre V1 y V2 del tipo $[ \pm$ pasado ... \pm pasado]. Por ejemplo:

Niego/negué rotundamente que sostenga/haya sostenido/sostuviera/hubiera sostenido vínculos con el Partido Nazi

Me alegra que te los agradecieran 
(13) Te aconsejé que te acostaras.

La oración (13) no realiza un acto directivo sino una aserción, es decir, la modalidad de fuerza ilocutoria depende del tiempo verbal. Si es un rasgo de $\mathrm{C}$, como propusimos anteriormente, debería establecerse cotejo/concordancia entre el $\mathrm{C}$ de la raíz y el mismo elemento de la subordinada, dos categorías funcionales idénticas serían sonda y meta ${ }^{17}$.

\section{El híbrido T-D}

En este punto es necesario considerar el aporte que realizan $\mathrm{T}$ y $\mathrm{D}$ a la interpretación de un enunciado.

En la sintaxis, ambos elementos actúan como variables, ya sea anafórica o deícticamente. Los tiempos verbales determinan cómo debe concebirse el evento temporalmente, $\mathrm{y}$ los determinantes establecen la referencia de las entidades mencionadas en el enunciado. En ambos casos, la referencia puede requerir recuperar un evento o entidad del discurso previo (anáfora), o bien recuperarlo del contexto de enunciación (deixis). En su condición de variables, deben estar ligadas a operador en el dominio inmediato, en el caso de las anáforas, o en el contexto, en el caso de los deícticos. (Chomsky, 1995).

17Podría plantearse que cotejo/concordancia se da entre los dos T, el resultado sería similar.

$N^{\circ}$ 7. Primer Semestre de 2017 
En (14) se trata de un pronombre interrogativo que liga sus copias y se identifica como el tema/objeto directo del verbo hendido; en (16) el D [+definido] entra en conflicto con los requerimientos del verbo 'querer' de la misma forma que se explicó en la sección precedente.

(14) ¿Quién quiere venir?

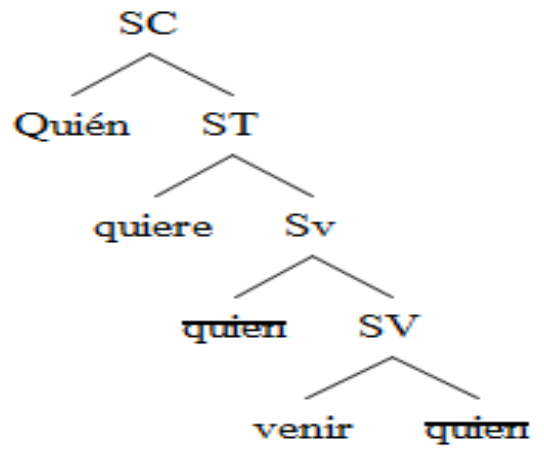

(16) Quiere una/*la mujer segura de lo que hace. 


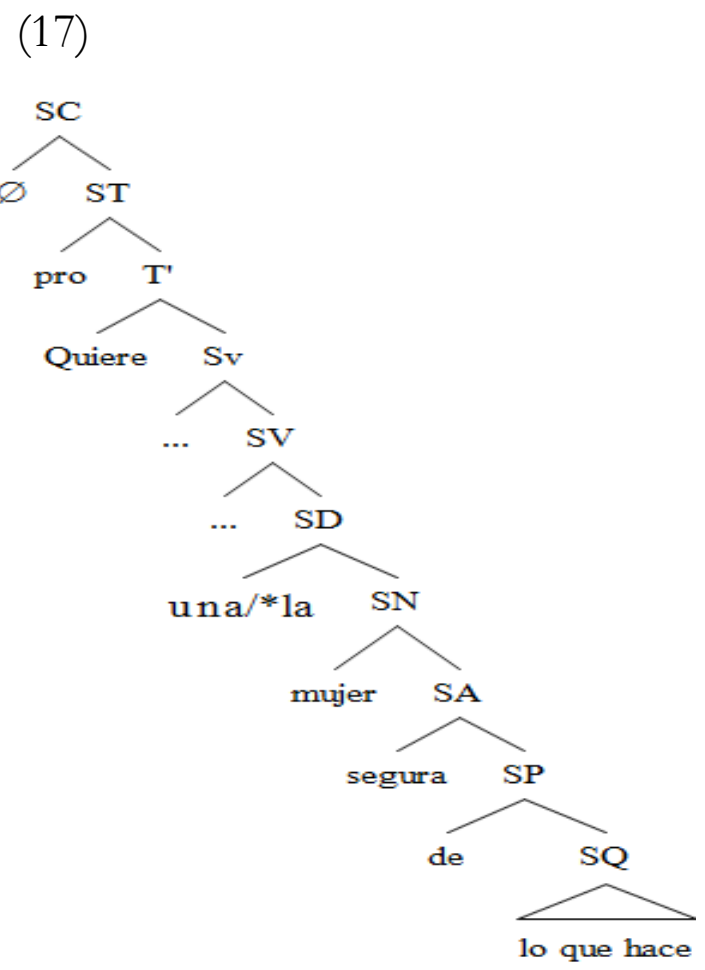

En tanto núcleos, D y T interactúan de forma análoga con sus complementos. En (18)-(21) Aktionsart y $\mathrm{T}$ entran en conflicto; en (18) el efecto es humorístico, en (19) la interpretación se obtiene por coacción. (20) y (21) resultan inaceptables porque la perfectividad del verbo matriz impide un verbo subordinado en modo subjuntivo:

(18) Supo ser arriero, pero después se olvidó. (Les Luthiers, 1979, canción 9).

(19) Supo entonces que nadie la esperaba;

(20) *Supo entonces que nadie la esperara/haya esperado; 
(21) * Sabe entonces que nadie la espere;

De la misma forma, (24) es inaceptable porque el clítico requiere que el complemento del verbo sea una cantidad [+específica], sin embargo, 'cerveza' es un $\mathrm{N}$ sin determinante y [-plural], con lo cual remite a una cantidad inespecífica.

(22) Se tomó la cerveza que había en la mesa (lectura no contable: "más de una botella", "toda la que había")

(23) Se tomó una cerveza que había en la mesa (lectura contable: "una botella")

(24) * Se tomó cerveza que había en la mesa.

Vemos en qué medida la cuantificación y [+/- especificidad] de entidades depende de D como la cuantificación de eventos depende de $\mathrm{T}$.

El ensamble de estas categorías en la sintaxis supone, entonces, no sólo la satisfacción de requisitos de localidad e interpretación de variables ligadas sino también la determinación de las variables con información contextual. Por lo tanto, y en tanto categorías procedimentales, $\mathrm{T}$ y $\mathrm{D}$ proporcionan instrucciones al módulo interpretativo para identificar las referencias de las entidades y/o estados o eventos del mundo.

Carston (2000) se refiere a este tipo de material lingüístico que requiere la 'saturación' de variables a partir de requisitos sintácticos como un 'híbrido' lingüístico/pragmático: 
...A level of communicated assumptions that are neither entirely controlled by linguistic semantics (logical form) nor are merely conversational implicatures; Recanati and Travis share with relevance theorists the view that this linguistic/pragmatic hybrid is what constitutes the truthconditional content of the utterance (p. 13).

Las instrucciones que activan $\mathrm{T}$ y $\mathrm{D}$ al ensamblarse en la sintaxis producen la "saturación" de las variables, entendida como el proceso por el cual se valúa una variable lingüística con información contextual para cumplir requisitos gramaticales. Desde la TR, es posible concebir este tipo de elemento lingǘstico a partir del Principio de Direccionalidad Lingüística, que determina que: "A pragmatically determined aspect of meaning is part of what is said iff its contextual determination is triggered by the grammar, that is, if the sentence itself sets up a slot to be contextually filled" (Carston, 2000, p. 17).

Es decir que la conformación de explicaturas y simultánea obtención de implicaturas requiere que el módulo interpretativo acceda a la sintaxis en aquellos lugares que estén 'indicados', no sólo por núcleos de fase, sino por el ensamble de cualquier categoría funcional/procedimental. El acceso del módulo interpretativo, entonces, puede considerarse 'irrestricto': cada CP activará procesos inferenciales que saturarán variables y enriquecerán/aflojarán conceptos según sea relevante a la obtención de una hipótesis interpretativa. 
Teniendo presente el paralelismo entre $\mathrm{T}$ y $\mathrm{D}$ ilustrado arriba, consideramos casos como el de (25) y (26), y sus estructuras en (27) y (28), a continuación:

(25) El corcho flota en el agua (lectura genérica)

(26) El corcho flotaba en el agua. (lectura individualizadora)

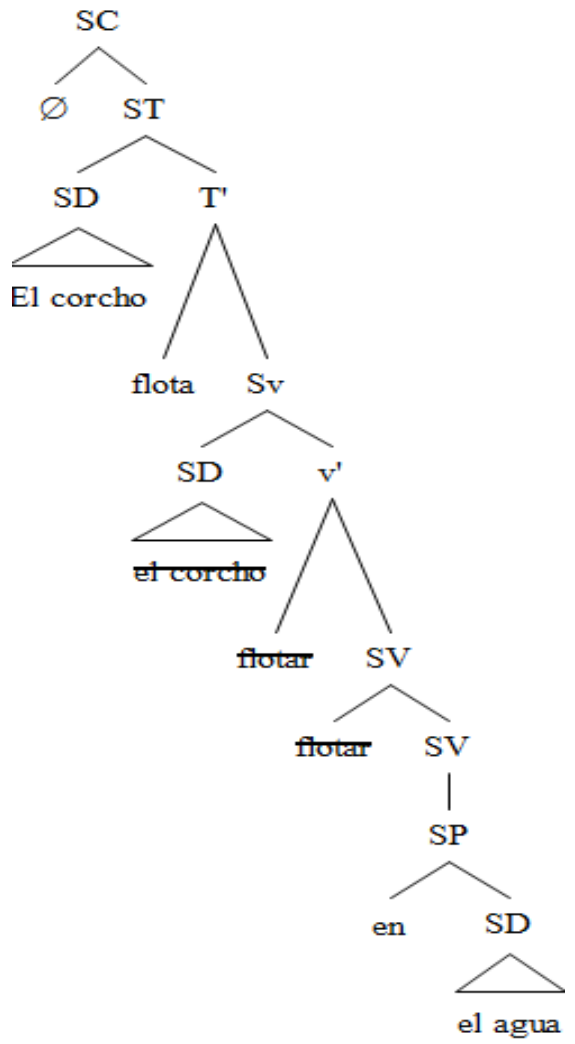




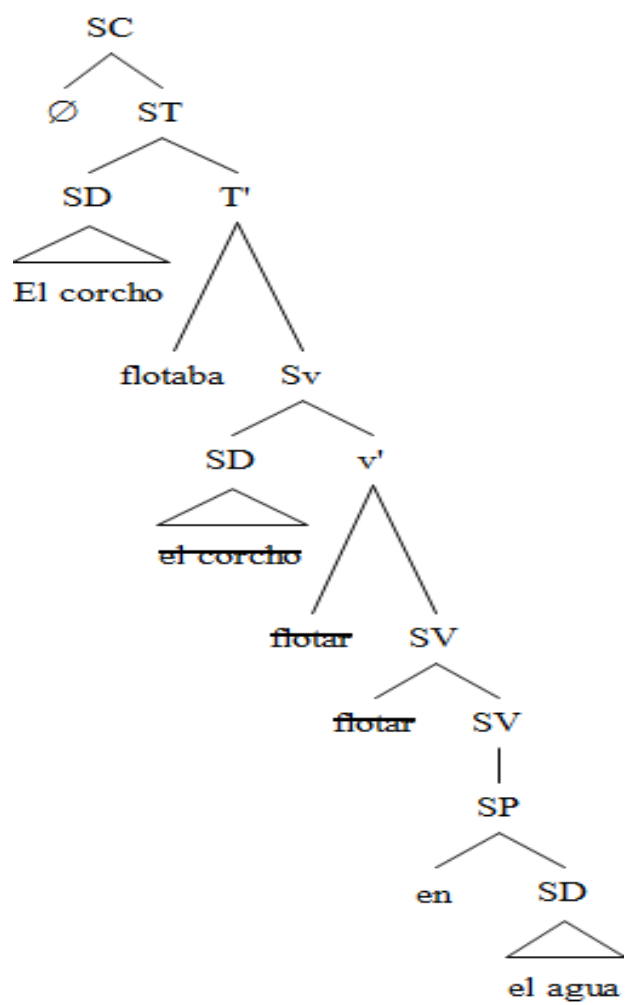

(29) Se aprueban leyes por mayoría simple. (lectura genérica)

(30) Se aprobaron leyes por mayoría simple. (Lectura individualizadora) 


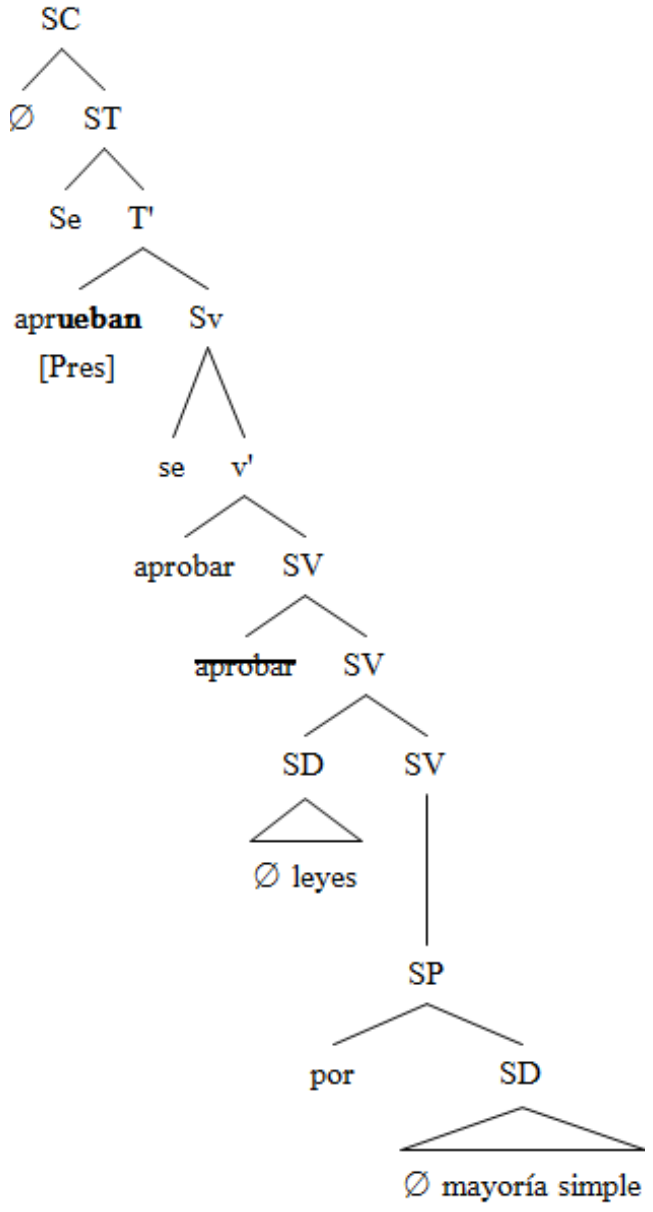



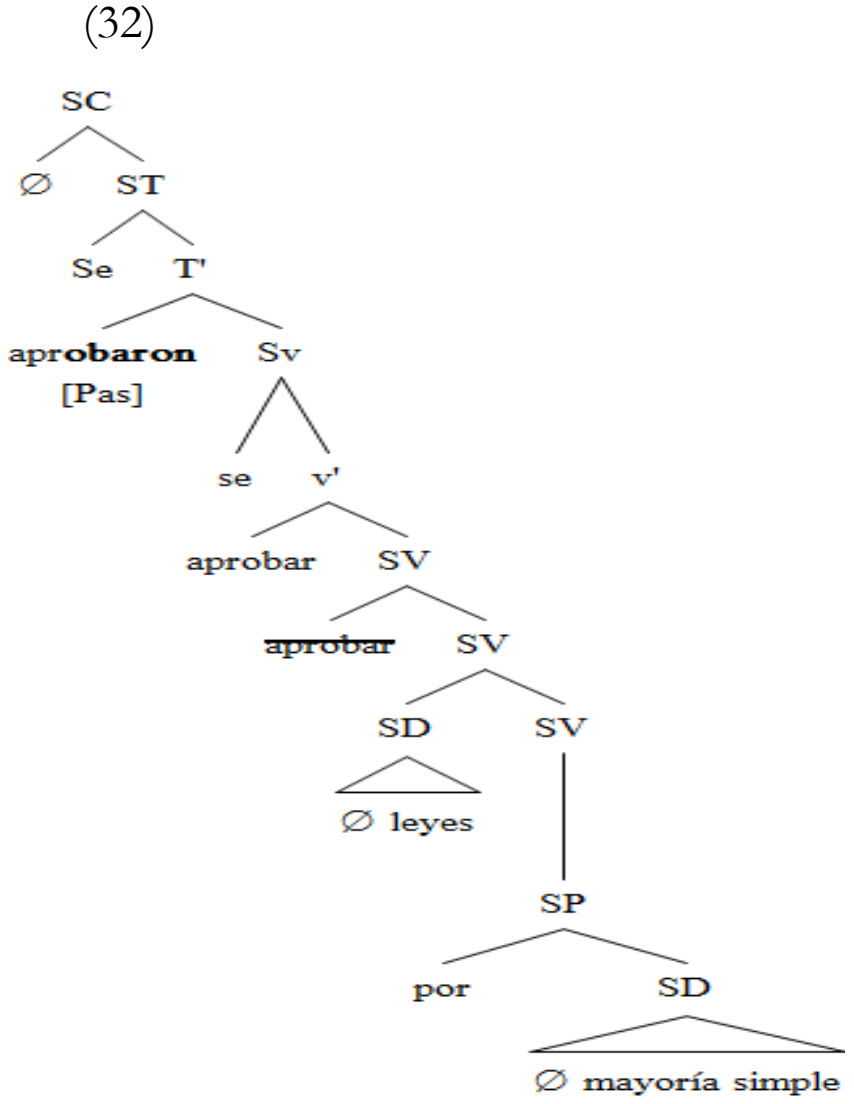

(33) Todos los estudiantes aprueban los exámenes sin dificultades. (lectura genérica)

(34) Todos los estudiantes aprobaron los exámenes sin dificultades. (lectura individualizadora) 
(35)

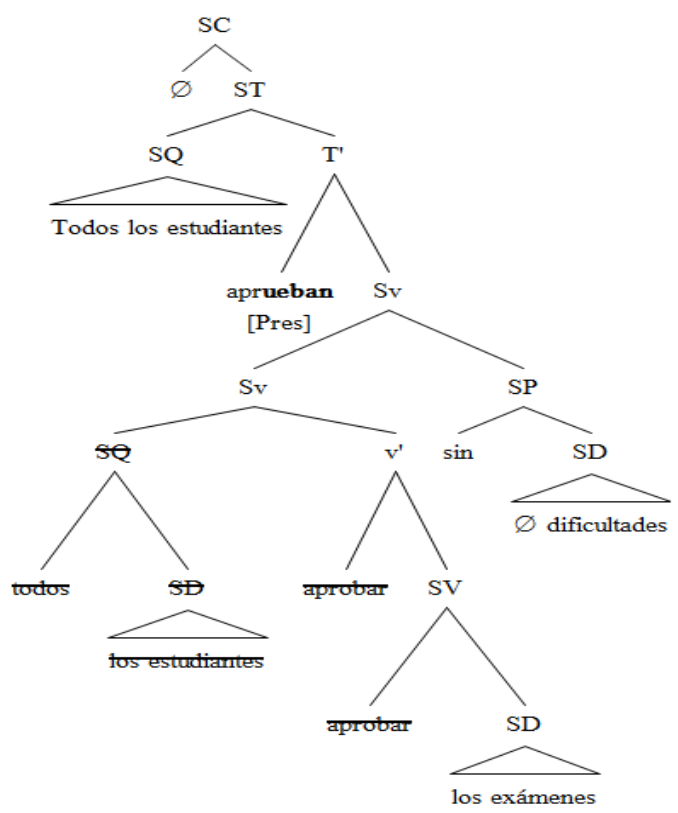

(36)

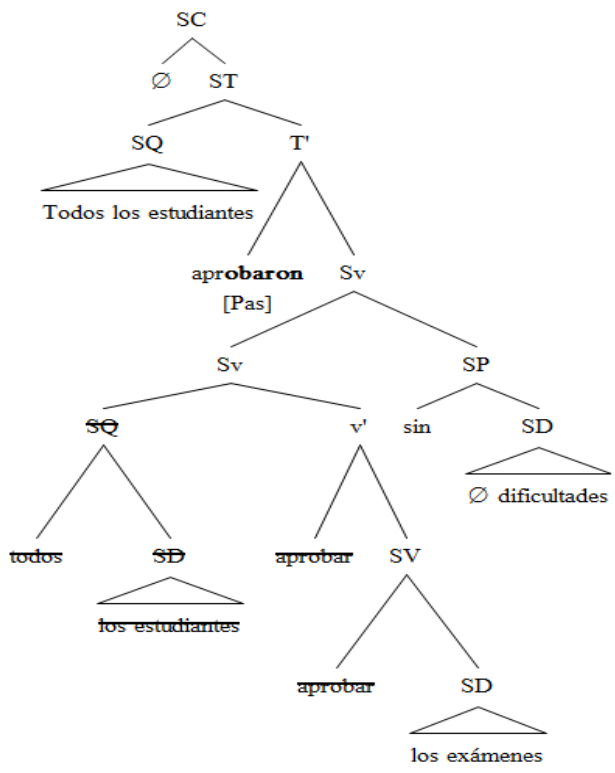


Como puede verse, es $\mathrm{T}$ el que determina si la lectura del argumento será genérica o individualizadora: el rasgo [+Pres] de $\mathrm{T}$ en (27), (31) y (35) condiciona que el rasgo de $\mathrm{D}$ sea [+definido/específico]; de forma análoga, el rasgo [-Pres] en (26), (30) y (34) determina un D con [-definido/específico].

Consideramos que casos como los expuestos en este trabajo y los análisis de Leonetti (2004) y Romero (2008) muestran que la saturación de $\mathrm{D}$ no es independiente de la saturación de T. Todo lo contrario. El cotejo y valuado de los rasgos [+/definido] o [+/-específico] no depende sólo de $\mathrm{D}$ sino que es necesario esperar a que se ensamble $\mathrm{T}$ para establecer la "saturación", que a su vez es un proceso que realiza el módulo interpretativo. Es decir que la saturación del híbrido T-D permite al módulo interpretativo completar las explicaturas (Sperber y Wilson, 1986), por medio de la valuación de las variables que se ensamblan en la sintaxis.

Es importante notar que el dudoso status de T como núcleo de fase y las consideraciones de Carston y otros respecto de ciertos aspectos gramaticales del significado que requieren saturación contextual nos lleva a pensar que tal vez es precisamente la condición de hibridez de T lo que dificulta su caracterización sintáctica, tal y como se analizó en Alabart Lago y Herrera (2013). Sostenemos que algo similar a lo que 
ocurre en T ocurre también con D: D es núcleo de fase pero no basta con que coteje sus rasgos de [especificidad/definitud]. $\mathrm{D}$ necesita de $\mathrm{T}$ ( $\mathrm{y}$, por tanto, del módulo interpretativo) para saturarse.

\section{Conclusiones}

En trabajos anteriores (Alabart Lago, Díaz y Herrera, 2012; Alabart Lago y Herrera, 2013), nos preguntamos si el hecho de que ciertas etapas de la interpretación de enunciados se correspondan en parte con fases en la derivación sintáctica implica que el módulo interpretativo opera derivacionalmente. Sabemos ahora que la respuesta es negativa. El módulo interpretativo accede a la sintaxis con cada ensamble de CCPP/CCFF: C, T, v y D (además de las CCFF de la periferia izquierda, como mostramos en Alabart Lago y Herrera (2013)). $\mathrm{El}$ acceso del módulo interpretativo a la sintaxis sería entonces "relativamente irrestricto" dado que depende de la aparición de una CP en la sintaxis.

En particular, el ensamble de $\mathrm{T}$ y $\mathrm{D}$ motiva el proceso inferencial de saturación de las variables con información contextual. Más aun, vimos que la saturación de D depende del ensamble de T. Esto nos lleva a proponer que más que el híbrido T (Carston, 2000), debemos considerar que ambas categorías constituyen un híbrido T-D que, al ensamblarse en la sintaxis, "llama" al módulo interpretativo para que complete las referencias con información contextual.

Vimos también que en los casos en que ciertos aspectos léxicos entran en conflicto con requerimientos del contenido 
procedimental, el módulo interpretativo siempre resuelve este conflicto en favor del requisito procedimental (mediante coacción o reajuste conceptual).

Existe un consenso (Chomsky, 2008) respecto de la inexistencia de niveles de representación (internos), ES, EP y en particular FL, a partir de consideraciones de localidad y economía. Siguiendo esta idea, entonces, consideramos que el proceso interpretativo, resultante de la interacción entre los módulos sintáctico e interpretativo, consta de:

- Materialización en la sintaxis y Transferencia de rasgos fonéticos a $\mathrm{FF}$.

- Acceso "relativamente irrestricto" del módulo interpretativo a la sintaxis, con cada ensamble de CCFF.

- Saturación y otros procesos inferenciales (aflojamiento/enriquecimiento, etc.) en el módulo interpretativo que está regido por los Principios de Relevancia.

Cuestiones que quedan pendientes

Las conclusiones que hemos extraído del análisis dejan pedientes de solución algunos problemas que sugieren posibles hipótesis de trabajo para futuras investigaciones.

FL no se considera como nivel de representación, por lo tanto no habría interfaz entre la sintaxis y CI, lo que ocasiona dificultades para la aplicación del CIF, el principio de economía aparentemente más importante propuesto por Chomsky (1993 y 1995). Sugerimos que es el "aparentemente más importante" porque otros principios de economía han 
sido descartados o unificados en análisis posteriores, por ejemplo "Último Recurso" y "Egoísmo/ Avaricia" (Greed). De todas maneras, Chomsky (2008) menciona en varias oportunidades que el contenido de las fases se transfiere a las interfaces, sin que se especifique claramente en qué consiste la interfaz con CI.

Ya se ha mencionado anteriormente que Recanati (2010) considera a las FFPP como "la interfaz entre el sistema lingüístico y el de pensamiento" porque estas representaciones son legibles para la sintaxis y el módulo interpretativo. En consecuencia, podría considerarse válido sugerir que las FFPP o explicaturas son las interfaces. Teniendo en cuenta que en TR se distinguen dos niveles de explicatura - la explicatura proposicional y la explicatura de nivel superior -, surge la idea de que la explicatura proposicional podría constituir la interfaz de la primera transferencia -la de la fase de Sv- y la explicatura de nivel superior -la de la segunda transferencia-, la de SC.

Esta propuesta tiene el inconveniente de que la explicatura proposicional requiere de la saturación de las variables temporales, con lo que no podría constituirse con los elementos transferidos por la fase de $\mathrm{Sv}^{18}$.

Una línea de investigación que podemos esbozar es la recuperación limitada de la noción de "fase fuerte" y "fase débil", dejada de lado en los últimos análisis generativistas. C y $\mathrm{v}$ serían núcleos de fase $\mathrm{y}$, una vez agotado el cotejo y valoración de rasgos, se transfiere su contenido. $\mathrm{D}$ y $\mathrm{T}$ pueden

18 Además, creemos que debe notarse que si la transferencia se realiza en este punto de la derivación muchos sujetos sintácticos no habrían aún cotejado y valorado su rasgo de Caso, dado que dicho cotejo se realiza mediante Ensamble Interno con T. 
ser considerados, dada su hibridez ya explicada, como estadios de la derivación a los cuales el componente interpretativo puede acceder en forma limitada para que los procesos inferenciales actúen y faciliten las operaciones sintácticas posteriores - cotejo y concordancia - y, lo más significativo a nuestro juicio, puedan evitar que rasgos no interpretables alcancen CI (o la interfaz). Esto, como ya se ha dicho, agregaría economía al sistema, dado que permitiría cancelar derivaciones sin que estas deban llegar a una fase superior para ser evaluadas.

\section{Referencias Bibliográficas}

Alabart Lago, L. y Herrera, G. (2013). La lengua en uso: Un fenómeno de interfaz. Ponencia presentada en las II Jornadas de Jóvenes Lingüistas (UBA), Buenos Aires. Recuperada de http://il.institutos.filo.uba.ar/sites/il.institutos.filo.uba.ar/fil es/Actas\%20II\%20Jornadas\%20de\%20Jo\%CC\%81venes\%20 Lingu\%CC\%88istas.pdf

Alabart Lago, L., Díaz, P. y Herrera, G. (2012). Adverbios Modalizadores: Una caracterización cognitiva. Ponencia presentada en el Congreso de la Delegación Argentina de la Asociación de Lingüística y Filología Hispánica de América Latina (ALFAL) y $\mathrm{V}$ Jornadas Internacionales de investigación en Filología Hispánica. UNLP. La Plata, Argentina. Recuperado de http://www.memoria.fahce.unlp.edu.ar/trab eventos/ev.101 4/ev.1014.pdf

Austin, J. (1964). Cómo hacer cosas con palabras. Buenos Aires: Paidós.

Boeckx, C., \& Grohmann, K. (2004). Barriers and phases: Forward to the past. En Tools in Linguistic Theory, 16-18. 
Budapest: Institute of Linguistics of the Hungarian Academy of Sciences \& Utrecht Institute of Linguistics OTS.

Carston, R. (2000). Explicature and semantics. UCL Working Papers in Linguistics, 12, 1-44.

Chomsky, N, (1966). Topics in the theory of Generative Grammar. La Haya: Mouton.

Chomsky, N. (1973). Conditions on transformations. En R. Anderson y P. Kiparsky (eds.) A Frestschift for Morris Halle. New York: Holt, Reinhart y Winston.

Chomsky, N. (1986). Barriers. Cambridge: MIT Press.

Chomsky, N. (1993). A Minimalist Program for Linguistic Theory. En K. Hale \& S.J. Keyser (eds.) The View from Building 20. Cambridge: MIT Press.

Chomsky, N. (1995). The Minimalist Program. Cambridge: MIT Press.

Chomsky, N. (1998). Minimalist Inquiries: The Framework. En MIT Ocassional Papers in Linguistics, 15. Cambridge: MIT Press.

Chomsky, N., Halle, M., \& Lukoff, F. (1956). On accent and juncture in English. En M. Halle et al. (eds) For Roman Jakobson, 65-80. La Haya: Mouton.

Chomsky, Noam (2001). Derivation by Phase. En M. Kenstowicz (ed.), Ken Hale A Life in Language 1-52. Cambridge (Mass.): MIT Press.

Chomsky, Noam (2008). On Phases. En C. Otero et al. (eds.) Foundational Issues in Linguistic Theory. Cambridge (Mass.): MIT Press.

Gallego, Á. y Uriagereka, J. (2006). Conditions on Subextraction. Conferencia presentada en el Coloquio de 
Gramática Generativa 16. Universidad Autónoma de Madrid. Recuperado http://filcat.uab.cat/clt/membres/professors/gallego/pdf/Ga llego Uriagereka CoS(draft).pdf

Gallego, Á. y Uriagereka, J. (2006). Sub-extraction from Subjects. En Actas de WCCFL 25. University of Washington.

Gallego, Ángel J. (2006). Phase Effects in (Iberian) Romance. Cuadernos de Lingüística del IUOG 13, 59-78. Instituto Universitario Ortega y Gasset de Madrid.

Gallego, Ángel J. (2007). Phase Theory and Parametric Variation. Tesis doctoral de la Universitat Autónoma de Barcelona.

Gallego, Ángel J. (2010). Phase Theory. Amsterdam: John Benjamins Publishing Co.

Huang, C.-T.J, (1994).Logical Form. En G. Webelhuth, (ed.) Government and Binding Theory and the Minimalist Program, 127-175. Oxford: Blackwell

Leonetti, M. (2004). Sobre tiempos y determinantes. Actas del V Congreso de Lingüística General. Madrid: Arco.

Leonetti, M. y Escandell Vidal, M. V. (2004). Semántica conceptual / Semántica procedimental. Actas del V Congreso de Lingüística General. Madrid: Arco.

Les Luthiers (1990). El explicado. En Mastropiero que nunca [CD] España: Ariola.

May, R. (1985). Logical Form. Cambridge: MIT Press.

Recanati, F. (2010). Truth-Conditional Pragmatics. Oxford: Oxford University Press.

Rizzi, L. (1990). Relativized Minimality. Cambridge: MIT Press. 
Romero, D. (2008). Fases en la derivación y categorías procedimentales. Presentado en el XI Congreso de la SAL, UNL. Recuperado de https://es.scribd.com/document/56263941/D-RomeroFases-en-La-Deriv

Searle, J. (1975). Indirect Speech Acts. En Cole, Peter \& Jerry L. Morgan, (eds.) Syntax and Semantics, 3, 59-82. New York: Academic Press.

Sperber, D \& D. Wilson (1986/95) Relevance: Communication and Cognition. Cambridge: Harvard University Press.

Suñer, M. y Padilla-Rivera, J. (1987). Sequence of Tenses and the Subjunctive, Again. En Hispania 70 (3), 634-642. 